\title{
Modular Extension of the ACR-NEMA DICOM Standard to Support New Diagnostic Imaging Modalities and Services
}

\author{
W. Dean Bidgood Jr and Steven C. Horii
}

The American College of Radiology-National Electrical Manufacturers Association (ACR-NEMA) Standard for Digital Imaging and Communications in Medicine (DICOM) (NEMA PS3-1993, DICOM) enables interconnection of imaging equipment and exchange of diagnostic images over standard networks. The widespread adoption of DICOM by industry and the benefits of standardized image management have attracted international attention. Other medical specialties, dentistry, and veterinary medicine have indicated strong interest in extending the DICOM Standard to support nonradiological image modalities. Parts 1 through 9 of DICOM were published in 1992 and 1993. Additional features developed in response to requests from the user community are being added in DICOM Supplements. The ACR, NEMA, and committees from other medical specialties working under the coordination of the American National Standards Institute Healthcare Informatics Standards Planning Panel Message Standards Developers Subcommittee Joint Working Group for Diagnostic Image Communication have defined new image object classes to support X-Ray Angiography, Radio-Fluoroscopy, Positron Emission Tomography, Endoscopy, and Light Microscopy. ACR-NEMA has also developed new service classes for Storage Commitment and Worklist Management. In joint work with the standards development committee of the Japanese Industries for Radiation Apparatus and the Japanese Medical Information System Development Center, ACR-NEMA has developed specifications for multibyte character set support and for a DICOM file format for transportable media. Other work is in progress. New DICOM features have provided and can continue to provide significant new functionality for digital image management systems. The demonstrated capability of efficient extension of the base standard is evidence of the effectiveness of the object oriented strategy of DICOM.

\section{Copyright $\odot 1996$ by W.B. Saunders Company}

KEY WORDS: Standard for Digital Imaging and Communications in Medicine (DICOM), American College of Radiology-National Electrical Manufacturers Association (ACR-NEMA), Health Level Seven (HL7), endoscopy, light microscopy, image management, information system.

T HIS IS an overview of recent additions to the ACR-NEMA DICOM Standard (the American College of Radiology, National Electrical Manufacturers Association Standard for Digital Imaging and Communications in Medicine). The list of imaging modalities and image management services supported by DICOM have grown considerably beyond the features described in our introductory DICOM articles in 1992.1,2 DICOM has become the de-facto international standard for interconnection of healthcare imaging equipment and exchange of diagnostic and therapeutic images of all imaging modalities. At the time of this writing, DICOM extensions have been proposed for nearly every imaging modality that is used in healthcare. The DICOM Standard derives its extensibility from its object oriented information model. The DICOM information model can be extended in logical modules where needed to support new technology. New features are added to the Standard in Supplements that define one or more new information objects, query models, encoding rules, or services.

Because data interchange standards are quasilegal documents on which engineering specifications and contracts are routinely based, the DICOM Standard and related healthcare data interchange standards are written in a concise, sometimes cryptic, formal style that unfortunately defies comprehension by casual readers. Acronyms are used liberally to reduce the bulk of the highly detailed technical specifications. Because articles about data interchange standards unavoidably reflect the jargon of the standards themselves we have included Table 1, a summary of abbreviations frequently encountered in the context of DICOM and related standards development activities, to facilitate the comprehension of DICOM idioms.

From the Division of Medical Informatics, Department of Community and Family Medicine, Duke University Medical Center, Chapel Hill, NC; and the Department of Radiology, Hospital of the University of Pennsylvania, Philadelphia.

Supported in part by the National Libran of Medicine, the American College of Radiology, Duke University, the University of Pennsylvania, the University of North Carolina at Chapel Hill, the University of Florida, the College of American Pathologists, and the European Committee for Standardization.

Address reprint requests to $W$. Dean Bidgood, MD, MS,

502/_ Dogwood Dr, Chapel Hill, NC 27516.

Copyright $\mathrm{C} 1996$ by W.B. Saunders Company

0897-1889/96/0902-0003\$3.0010 
This report is an update of the two-part standard for DICOM overview that we published in 1992.1.2

\section{Multispecialty DICOM Development}

Since 1982, the ACR and the NEMA have worked jointly in the ACR-NEMA Committee to develop the DICOM Standard. ${ }^{3}$ In the early years of ACR-NEMA development, our work was focused on Radiology imaging. ${ }^{4}$ However, the transformation of the ACR-NEMA version 2.0 standard to DICOM included generalization of the specification to allow definition of Image Objects and Service Classes to support any type of imaging modality. The original release of DICOM (Parts 1 through 9) is the base standard for network image management. Parts 10 through 12, published as DICOM Supplements, allow DICOM messages to be stored and managed as files on transportable media. The DICOM base standard (Parts 1 through 12) provides a general technical foundation for description of any type of diagnostic or therapeutic image. The base standard thus provides a set of image management tools that greatly simplifies and streamlines the development of a compatible set of generic image communication formats for not only radiographs and other radiological image modalities, but for any type of diagnostic or therapeutic image.

The extension of DICOM beyond Radiology began in 1992. In March of that year, one of the authors (W.D.B.) presented an overview of the draft DICOM Standard to the American College of Cardiology (ACC) ad hoc Committee for Digital Imaging in the Coronary Catheterization Laboratory. Although at first inclined to pursue an independent standards development effort, the ACC at that meeting decided to consider the benefits of adding components to the DICOM Standard rather than creating a new standard from the ground up. The ACR, ACC, and NEMA met again in October 1992 and in January 1993 to organize a joint development effort. The resulting standard for X-Ray Angiography (XA) passed final ballot in 1995. The similarity of basic elements of the DICOM XA image communication to the original set of DICOM radiological image types enabled industry to rapidly develop prototypes (before the final ballot of the Standard) and to bring products to market immediately after publication of the XA Supplement.

In anticipation of similar adaptation of DICOM for use by other specialties, the ACRNEMA Committee formed a new Working Group (ACR-NEMA WG-9) in 1992, to coordinate with other standards developing organizations and to facilitate multispeciality participation in DICOM development. ACR-NEMA in 1992 also became a founding member of the American National Standards Institute Healthcare Informatics Standards Planning Panel (ANSI HISPP), an organization formed by ANSI to coordinate United States standards for healthcare informatics. In June 1992, the ANSI HISPP Message Standards Developers Subcommittee (MSDS) delegated the coordination of diagnostic image communication standards to ACRNEMA. Shortly after this, ACR-NEMA WG-9 met with delegates from medical professional societies and standards developing organizations (SDO's) to form the ANSI HISPP Joint Working Group for Diagnostic Image Communication (DJWG). The DJWG met for the first time in January 1993. Later that month, one of the authors (W.D.B.) presented an overview of DICOM to the board of the American Society for Clinical Pathologists (ASCP). The ASCP board voted to adopt the DICOM Standard as the basis for Pathology image communication. In mid-1993, the American Society for Gastrointestinal Endoscopy (ASGE) also adopted the DICOM Standard. By 1994, the number of organizations that had either adopted DICOM or had indicated interest in participation in development of specialized extensions of the DICOM Standard had grown to nearly 20 . Table 2 lists the names of organizations that have participated in the DJWG.

The DJWG initiated the formation of ad-hoc standards development committees for Gastrointestinal Endoscopy and for Pathology imaging communication. The ASGE has taken the initiative of developing an extension of the DICOM Standard for endoscopy. ${ }^{6}$ This work has been done in liaison with other medical specialty societies that use endoscopic, laparoscopic, and surgical microscopic techniques. A public dem- 
Table 1. Abbreviations and Acronyms Pertaining to the DICOM Standard and Related Standards Development Activities

\begin{tabular}{|c|c|}
\hline $\mathrm{ACC}$ & American College of Cardiology \\
\hline ACR & American College of Radiology \\
\hline ACR-NEMA & $\begin{array}{l}\text { American College of Radiology-National Electrical Manufacturers Association Digital Imaging and Commu- } \\
\text { nications Standards Committee }\end{array}$ \\
\hline ACR-NEMA WG6 & $\begin{array}{l}\text { Base Standard Working Group of the American College of Radiology-National Electrical Manufacturers } \\
\text { Association Digital Imaging and Communications Standards Committee }\end{array}$ \\
\hline ACR-NEMA WG9 & $\begin{array}{l}\text { Standards Harmonization Working Group of the American College of Radiology-National Electrical Manufac- } \\
\text { turers Association Digital Imaging and Communications Standards Committee }\end{array}$ \\
\hline ADT & Admission, Discharge, Transfer \\
\hline ANSI & American National Standards Institute \\
\hline ASGE & American Society for Gastrointestinal Endoscopy \\
\hline BSI & British Standards Institute \\
\hline CAP & College of American Pathologists \\
\hline CAP-IEC & College of American Pathologists-Image Exchange Committee \\
\hline CEN & European Standardization Committee \\
\hline CEN/TC 251 & European Standardization Committee. Technical Committee for Medical Informatics \\
\hline CEN/TC251/WG3 & $\begin{array}{l}\text { European Standardization Committee. Technical Committee for Medical Informatics. Working Group 3: } \\
\text { Healthcare Communications and Messages }\end{array}$ \\
\hline CEN/TC251/WG4 & $\begin{array}{l}\text { European Standardization Committee. Technical Committee for Medical Informatics. Working Group 4: } \\
\text { Medical Imaging and Multimedia }\end{array}$ \\
\hline CDM & Common Data Model \\
\hline CDR & Common Data Repository \\
\hline CPRS & Computerized Patient Record System \\
\hline CDT & Common Data Types \\
\hline CPU & Central Processing Unit \\
\hline CT & Computed Toinography \\
\hline CU! & UMLS Unique Concept Identifier \\
\hline DICOM & Digital Imaging and Communications in Healthcare \\
\hline DICOMDIR & DICOM File Directory Services \\
\hline $\mathrm{DIM}$ & Domain Information Model \\
\hline DIMSE & DICOM Message Service Element \\
\hline DJWG & ANSI HISPP MSDS Joint Working Group for Diagnostic Image Communication \\
\hline DSG & DICOM Service Group \\
\hline EIMEX & Endoscopic Image Exchange Committee \\
\hline EMR & Electronic Medical Record \\
\hline EMRS & Electronic Medical Record System \\
\hline FSC & DICOM File Set Creator \\
\hline FSR & DICOM File Set Reader \\
\hline FSU & DICOM File Set Updater \\
\hline HIS & Hospital Information System \\
\hline HISPP & ANSi Healthcare Informatics Standards Planning Panel \\
\hline $\mathrm{HL7}$ & Health Level Seven \\
\hline ID & Identification \\
\hline IEEE & Institute of Electrical and Electronics Engineers \\
\hline IEEE P1157 & Trial-Use Standard for Health Care Data Interchange-Information Model Methods: Data Model Framework \\
\hline IMACS & Image Management and Communication System \\
\hline IMSIG & HL7/DICOM Image Management Special Interest Group \\
\hline ISIS & Information System-Imaging System \\
\hline IOD & Information Object Definition \\
\hline !SO & International Standards Organization \\
\hline IST & Institute of Standards and Technology (United Kingdom) \\
\hline IS\&C & Image Save and Carry \\
\hline LIS & Laboratory Information System \\
\hline JAHIS & Japanese Association for Hospital Information Systems \\
\hline JIRA & Japan industries Association of Radiation Apparatus \\
\hline JWG & Joint Working Group \\
\hline JWG-CDM & Joint Working Group for a Common Data Model \\
\hline JWG-CDT & Joint Working Group for Common Data Types \\
\hline MEDICOM & Medical Image Communication \\
\hline $\mathrm{MeSH}$ & Medical Subject Headings \\
\hline
\end{tabular}


Table 1. Abbreviations and Acronyms Pertaining to the DICOM Standard and Related Standards Development Activities (Cont'd)

\begin{tabular}{|c|c|}
\hline MIPS & Medical Image Processing Standard \\
\hline MRI & Magnetic Resonance Imaging \\
\hline MSDS & ANSI HISPP Message Standards Developers Subcommittee \\
\hline NEMA & National Electrical Manufacturers Association \\
\hline NLM & (United States) National Library of Medicine \\
\hline OOA & Object Oriented Analysis \\
\hline PACS & Picture Archiving and Communication System \\
\hline PT3-018 & $\begin{array}{l}\text { European Standardization Committee. Technical Committee for Medical Informatics: Working Group 3: } \\
\text { Healthcare Communications and Messages. Project Team 18: Recording Data Sets used for Information } \\
\text { Interchange in Healthcare }\end{array}$ \\
\hline PT3-022 & $\begin{array}{l}\text { European Standardization Committee. Technical Committee for Medical Informatics: Working Group 3: } \\
\text { Healthcare Communications and Messages. Project Team 22: Request and Report Messages for Diagnostic } \\
\text { Services Departments }\end{array}$ \\
\hline PT4-020 & $\begin{array}{l}\text { European Standardization Committee. Technical Committee for Medical Informatics: Working Group 4: } \\
\text { Medical Imaging and Multimedia. Project Team 20: Medical Data Interchange: HIS/RIS/PACS and HIS/ } \\
\text { RIS-Modality Interfaces }\end{array}$ \\
\hline QA/DM & Quality Assurance/Data Modeling \\
\hline RIS & Radiology Information System \\
\hline SC & Service Class \\
\hline SCP & Service Class Provider \\
\hline SCU & Service Class User \\
\hline SDM & SNOMED DICOM Microglossary \\
\hline SDO & Standards Developing Organization \\
\hline SNOMED & $\begin{array}{l}\text { Systematized Nomenclature for Human and Veterinary Medicine (College of American Pathologists and the } \\
\text { American Veterinary Medical Association) }\end{array}$ \\
\hline SOP & Service Object Pair \\
\hline SOPC & Service Object Pair Class \\
\hline TC & Technical Committee \\
\hline UID & Unique Identifier \\
\hline UMLS & Unified Medical Language System (U.S. National Library of Medicine) \\
\hline WG & Working Group \\
\hline
\end{tabular}

onstration of DICOM endoscopic image communication is planned for 1996. The College of American Pathologists (CAP) has begun the development of a family of DICOM conformant standards for all types of Pathology image modalities. The CAP has formed an ad-hoc Image Exchange Committee (the CAP-IEC) to complete this project. The CAP-IEC met for the first time in November 1994, to begin development of a DICOM specification for exchange of light microscopy digital images.

\section{The Information System-Imaging System (ISIS) Interface}

In addition to binary image pixel data, Imaging Systems must handle a subset of imagerelated text information. Imaging Systems* typi-

*The term Imaging Systems is used to represent the computer systems that are primarily concerned with the acquisition, processing, transmission, archival, and presentation of binary image pixel data produced by various clinical image modalities (such as computed tomography [CT], cally must obtain this image-related information via queries to Information Systems $\dagger$ that do not support the DICOM Standard. The domain of

magnetic resonance imaging [MRI], Ultrasonography, Microscopy, Endoscopy), and by in vitro laboratory procedures (such as Western blot tests, Chromatography, Colorimetry, Tissue Culture, and Microbiological imaging). Imaging Systems are also known as communication systems or image management and communication systems.

$\uparrow$ The capitalized form, Information System, is used to represent the primarily text-based computer systems that support administrative, clinical, and financial information management in medical facilities—such as hospital information systems, laboratory information systems, radiology information systems, and computerized patient record systems. These are information systems that typically have been designed to support the functions of service request (order) and service report (result), with emphasis on capture of data for admission/discharge/transfer, scheduling, patient tracking, document production, and billing. The restriction of the term Information System to the definition specified in this paragraph makes sense in the context of the DICOM Standard, because the restricted meaning is in common use and is understood correctly by healthcare information system managers and developers. 
Table 2. Organizations That Have Participated in the ANSI HISPP MSDS Joint Working Group for Diagnostic

Image Communication

\begin{tabular}{lll}
\hline Cardiology & ACC & American College of Cardiology \\
Dentistry & ADA & American Dental Association \\
Dermatology & AAD & American Academy of Dermatology \\
Gastroenterology & ACG & American College of Gastroenterology \\
& AGA & American Gastroenterological Association \\
& ASGE & American Society for Gastrointestinal Endoscopy \\
General Surgery & ESGE & European Society for Gastrointestinal Endoscopy \\
Obstetrics and Gynecology & ACS & American College of Surgeons \\
Oral/Maxillofacial Radiology & ACOG & American College of Obstetrics and Gynecology \\
Pathology & AAOR & American Association of Orat-Maxillofacial Radiology \\
& ASCP & American Society of Clinical Pathologists \\
Pulmonology & CAP & College of American Pathologists \\
Radiology & ACCP & American College of Chest Physicians \\
Standards Developing & ACR & American College of Radiology \\
Organizations & ASTM & American Society for Testing and Materials \\
& HL7 & Health Level Seven \\
& IEEE & Institute of Electrical and Electronics Engineers \\
& NCPDP & National Council of Prescription Drug Providers \\
Urology & X12-N & Electronic Document Interchange Standards for Banking and Industry \\
Veterinary Medicine & AUA & American Urological Association \\
& AAVM & American Association of Veterinary Medicine \\
& AVMA & American Veterinary Medical Association \\
\hline
\end{tabular}

image-related text information is the subject of considerable interest among standards developing organizations who develop specifications for ISIS interfaces. The functions that must be supported by an ISIS interface include requestreport management, examination protocol management, procedure scheduling, resource management, query-retrieval, status reporting, event notification, charge capture, accountability, precertification, quality control, outcomes analysis, decision support, and others. The following SDO's have published the most widely implemented standards for these functions: (1) $A C R$ $N E M A$, (2) The European Committee for Standardization-Technical Committee for Medical Informatics Standards (CEN/TC251), and (3) Health Level Seven (HL7). However, currently no single standard supports the full list of functions needed for the ISIS interface and the existing versions of the most widely implemented standards are not fully equivalent in the areas of functional overlap.

Major standards development projects related to the ISIS interface are currently ongoing internationally in the private sector and in the public sector to improve the definition of (and to enable standard implementation of) the ISIS Interface. ${ }^{7-17}$ The ISIS Project, begun in 1995, involves ACR-NEMA WG9 and WG6, CAP-
IEC, HL7, CEN/TC251/WG4, CEN/TC251/ WG3 and members of other standards development organizations. ${ }^{18-25}$ The ISIS Project is an initiative to define the ISIS Context Model, an information model for the ISIS Interface, based on a mapping of the information models of the leading standards in the ISIS domain. One of the goals of the ISIS Project is to evolve the ISIS interface specifications of the related standards toward a common information model (also known as a Domain Information Model that supports the concepts contained in the ISIS Context Model. The ISIS Context Model is a merger of DICOM, PT4-020, CAP-IEC, and PT3-022 information models plus additional object classes that appear in none of the existing models.

\section{Worklist Management}

The DICOM Basic Worklist Management Service Class defines a generic worklist service. ${ }^{26} \mathrm{~A}$ worklist is a template that specifies the information that is needed to fulfill a defined set of tasks. Necessary details about the tasks (such as the order of fulfillment or properties of items involved in the tasks) are entered as values in the template. The Service Class also defines the message protocol to be used for the communication of worklist messages between information 
systems. Based on the generic template and protocol, the DICOM Basic Worklist Management Service Class defines an imaging Modality Worklist. The Modality Worklist allows an Information System to send a list of patients, examination times, and other information about the patients or examinations to the operator's console of a particular CT or MRI scanner.

The DICOM Modality Worklist Service Object Pair Class (SOPC) defines a new class of DICOM Composite Objects-the Modality Worklist Information Object Definition (IOD). A Modality Worklist is represented as a list (an ordered set) of items. Each item in the list is a Modality Worklist Object (a document that inherits values from multiple classes of real world objects-Scheduled Procedure Step, Requested Procedure, Imaging Service Request, Facility Episode, and Patient). The imaging modality operator obtains a Modality Worklist by initiating a query message to the Information System that manages the desired information. The query keys and the semantics for the values to be returned are defined by the DICOM Query/Retrieve information model. In the future, the generic worklist template and service may be specialized for various other contexts. For example, worklists may be defined to list the completed studies that are ready for interpretation by a particular subspecialist or to list the names and locations of patients that must receive oral contrast agent before their examinations.

\section{Storage Commitment}

The DICOM Storage Commitment Service Class provides a mechanism for one device to request that another device commit to store a set of images and related information according to previously defined set of parameters. ${ }^{27}$ The Storage Commitment Service Class significantly augments the existing Storage Service Class by defining a protocol for image management devices to share responsibility for storing and protecting a set of DICOM images and related information. The new Service Class helps to ensure that diagnostic images will not be deleted unexpectedly before intended retrieval.

The Storage Commitment Service Class applies to the application layer of the DICOM image management network. The protocol sup- ports two general scenarios. In the first scenario, the "Push" model, device-1 sends "pushes" a set of images to device- 2 and then requests confirmation of storage commitment from device- 2 regarding the previously-transmitted images. This model imposes relatively little additional processing overhead on image acquisition devices. In the second scenario, the "Pull" model, device- 1 notifies device- 2 that a set of images is available for storage. If device- 2 is able to offer storage commitment, then it retrieves "pulls" the images from device- 1 and reports the status of its operations to device-1, once it believes that processing is complete. In the event that errors occur, a protocol is defined for orderly recovery and appropriate notifications.

The DICOM Storage Commitment Service Class neither requires nor supports real time negotiation of storage and retrieval parameters at the time of association establishment. However, the storage parameters and the operational behavior of DICOM Storage Commitment Service Class Provider (SCP) and Service Class User application software must be declared explicitly in DICOM Conformance Statements. Documentation of storage parameters may include "under what conditions the SCP would delete SOP Instances; persistence of storage; capacity; volatility; and other pertinent information." Documentation of retrieval parameters may include "supported query/retrieve services; latency; and other pertinent information." Potential purchasers of such software have the opportunity to request functional parameters that meet their estimated needs. Vendors are able to express the functionality of their systems and to enter into purchase commitments with buyers according to clearly stated functional requirements. The Storage Commitment Storage Class satisfies a set of immediate functional needs and is the first step toward definition of a comprehensive DICOM Archive Management Service Class.

\section{Transportable Media: \\ DICOM Media Interchange}

Some applications in medical imaging require exchange of image and related information on removable media rather than over a network. Working Group V of ACR-NEMA in the mid 
1980's defined a magnetic tape format for exchange of ACR-NEMA Version 1 messages. In 1993, user requests for extension of the DICOM Standard to support file exchange on newer media prompted the reactivation of $W G \mathrm{~V}$. These requirements were based in part on the collaborative effort with the ACC as described in a previous section of this report. Early concepts of the strategy for supporting media interchange of DICOM Information Objects were developed by Charles Parisot of General Electric Medical Systems and reviewed by Dr Osman Ratib of the University of Geneva. From these early concepts, the strategy evolved to develop a set of logical layers that would coordinate with those used for network communication. The concept of supporting media exchange of DICOM Information Objects was envisioned in a three-layered model. The upper layer uses the IODs already developed and adds a file format and directory. The middle layer is the media format layer that describes the file system particulars to be used. Finally, the lower layer specifies particular media and the physical format of the media (such as 3.5-inch magnetooptical disk or recordable Compact Disc).

A new Part of DICOM, Part 10, describes Media Storage and File Formats. This Part is a media-independent set of definitions. It describes both a generic file and directory structure and a basic DICOM file service. This file service provides the connection to actual computer file systems and is analogous to the Generic Upper Layer Service of the network communication part of the Standard. The file service operates at a low level of media interchange. At a higher level, media interchange requires a set of services analogous to the DICOM Message Service Element services defined for network exchange of DICOM messages. An addendum to Part 4 defines the Media Storage Service Class that establishes three basic roles for DICOM devices that handle media. These are: File Set Reader, File Set Creator, and File Set Updater. Devices may support one or more of these roles. For example, it is possible to define DICOM media interchange devices that only read or only create files on media. The file format portion of Part 10 describes how to put a DICOM Data Set into a file along with a directory to point to the contents of such files. The file format includes an area called a File Preamble that is seen first by a device reading the file. This area is provided for potential compatibility with nonDICOM file services. In effect, a DICOM file may have "dual personalities." If read by a DICOM file reader, the File Preamble would be ignored and the remainder of the file read as a DICOM file. If read by a non-DICOM reader, that reader could look at the preamble for a pointer to information on how to read the DICOM file into its own file structure.

Each application that needs to record files onto a medium may require different media. For example, cardiologists need a high-capacity medium with fast access as a replacement for 35 $\mathrm{mm}$ cine film. However, noncardiac ultrasound specialists would probably not need as high a capacity, though they might need cine loop capability. For this reason, Parts 11 and 12 of DICOM serve to define further the media interchange support of DICOM all the way to the media level.

Each application will have an Application Profile (annexes of Part 11) that is a vertical "slice" through all the layers of DICOM. In effect, it will specify for a given application, the SOP classes, transfer syntax, directory structure, basic file service, media format, and physical medium needed. These profiles are necessary in part because, unlike communication over network connections, off-line communication by media inhibits the negotiation process. The media formats and physical media that are used for the standard are described in Part 12. The media format includes a description of the file system used. The file systems are, in some cases, particular to certain media. The PC file system is specified for most media as it is widely available on many different types of computers. However, for a recordable compact disc (CD-R) a file system specific to that medium, the ISO 9660 Standard, is required. Current media supported include CD-R, the $90 \mathrm{~mm}$ (3.5-inch) and $130 \mathrm{~mm}$ (5.25-inch) magneto-optical disks, and the $90 \mathrm{~mm}$ (3.5-inch) high-density floppy diskette (the latter intended primarily for testing and demonstrations). At the 1995 ACC Annual Meeting, prerecorded compact discs adhering to DICOM Parts 10,11, and 12 and containing DICOM XA and ultrasound information ob- 
jects were successfully shown as storage and exchange media. ${ }^{28}$

\section{Multibyte Character Sets}

In the process of working with the Japanese Medical Information System-Development Center (MEDIS-DC) Ad Hoc Committee members (Japanese Industries for Radiation Apparatus [JIRA] JAHIS, MEDIS-DC, IS\&C Committee) on the development of a Japanese standard for secure media interchange (called the Type 2 Standard), the question was raised as to how to handle the Japanese character set. The Type 2 Standard uses DICOM Information Objects and DICOM Part 10, but has a file system and media that follow the Image Save and Carry standard to provide the security functions required by the Japanese Ministry of Health and Welfare for recording and reading medical information on media.

The character set problem is that Japanese and other Asian languages that are based on pictographic characters have far more characters than can be represented with the 8 bits defined for most phonetic character sets (such as ASCII). Because the ACR-NEMA Committee thought that the Japanese have a far better understanding of the issues involved in representing their language than any organization in Europe or the United States, the task of developing an extension to DICOM that would support multibyte character sets was, with their approval, delegated to JIRA. Through exchanges of documents, consultation with European Standardization Committee members, and three joint meetings between JIRA and ACRNEMA, an extension to DICOM was developed over a 5 -month period. As of this writing, the extension is being balloted. When passed, it will become an addendum to Part 5 of DICOM.

JIRA used existing ISO standard methods for developing their extension as these are currently used in Japan. The Japanese phonetic character sets (Katakana, Hiragana) can be represented with a single byte. An ISO standard escape mechanism is used to specify the shift to two bytes for the pictographic (Kanji) character set. An interesting question was raised by the Japanese that had to do with representation of a patient's family name. This is typically represented with Kanji characters, but the Japanese pointed out that computer searches are much more efficient for phonetic representations. For this reason, the patient name (and other nametype elements) can be represented in two characters sets in the same DICOM message as a part of this extension. That also means that a system that can only understand ASCII could still display these name-type elements in readable form (and ignore the extended character set representation). To maintain alignment with other message standards, the dual representation of the patient name has been implemented by ACR-NEMA as an extension of the ANSI HISPP MSDS Person Name Common Data Type. ${ }^{29,30}$ As of this writing, the HL7 organization is considering the adoption of compatible specifications.

\section{Other Work in Progress}

Lossy compression. Ever-increasing image matrix sizes along with larger proportions of medical imaging done digitally have resulted in massive volumes of data that need to be transmitted and stored in a PACS. Data compression methods can reduce this volume, but to have a major impact, would have to go beyond the typical factor of 2 to 3 reduction that lossless compression methods yield. Lossy compression can often result in 10 to 1 compression or better. There are, naturally, concerns about the effects this would have on the image quality in terms of diagnostic content. To help determine if there is a lossy compression method that is suitable for diagnostic images at useful compression ratios, ACR-NEMA Working Group IV developed a request for proposals (RFP) that sought submissions of algorithms from industry and academia. The goal is to select a set of lossy compression techniques that could be incorporated into DICOM. The RFP was distributed in June 1995 to NEMA MedPACS members, ACR-NEMA Committee members, and was made available generally on the Internet. The RFP included proposed test methodology. A set of test images will be made available to those who wish to submit responses. The submission deadline was September 15, 1995 and by that time several responses had been received.

Perceptual linearization. A major problem for PACS that rely on cathode-ray tube displays is how to assure that an image displayed on one 
workstation will appear the same to an observer as that same image displayed on a different workstation. Unless there is a method by which this can be done, it will be very difficult to guarantee that image quality is being maintained. Photometric methods can be used to give uniformity, repeatability, and compensate for tube aging, but to be sure that a perceived image is consistent across workstations from different manufacturers or even including film displays, a method based on perceptual linearization is needed. ${ }^{31,32}$ Such a method assures that for a given change in digital input value to a monitor (or a laser film printer) a uniform perceived brightness (or optical density) change will be detected by an observer.

A new ACR-NEMA Working Group, WG $\mathrm{XI}$, was formed to develop a procedural standard for perceptual linearization of display devices. This is also referred to as the display function standard. This standard will not be part of DICOM, though users would certainly be encouraged to employ it as it is complementary to the use of DICOM. Unlike DICOM, this standard will be a procedural standard. The perceptual linearization technique involves characterizing a display and producing a lookup table that will map digital input levels to perceptual contrast levels. The perceptual contrast is modeled on human visual contrast sensitivity. It is important to note that resulting displays are not necessarily optimum in a diagnostic accuracy sense, but they are perceptually consistent across a wide variety of displays. It is anticipated that a draft of this standard will be available for comment in early 1996.

DICOM Lexicon: The SNOMED DICOM microglossary (SDM). The DICOM Image Object Classes for XA, Endoscopy, and Light Microscopy (as well as Nuclear Medicine, Ultrasound, and other existing DICOM image classes) have a common requirement for encoding anatomic concepts. To maintain consistency throughout DICOM, ACR-NEMA WG6 has adopted the strategy of referencing an external lexicon rather than defining ad hoc term lists for DICOM. DICOM messages reference terms from an external lexicon through the mechanism of triplet encoded sequence data ele- ments. $\ddagger$ Triplet encoded sequence data elements contain the unique identifier of a coding scheme (an external lexicon), a code value from the coding scheme, and an optional code meaning (a text label for the code value). ACRNEMA has selected the SNOMED International (CAP, Northfield, IL; American Veterinary Medical Association, Schaunburg, IL) lexicon as the default lexicon for DICOM triplet encoded sequence data elements. Of all lexicons evaluated by ACR-NEMA for this purpose, SNOMED has the most comprehensive listing of anatomic concepts. Also, because SNOMED is included in the Unified Medical Language System (UMLS) Metathesaurus of the United States National Library of Medicine (Bethesda, MD), information retrieval is improved by linkage to other lexicons, including the Medical Subject Headings. ${ }^{33,34}$

New triplet encoded sequence data elements have been defined to represent anatomic concepts drawn from the external lexicon. The new data elements are Anatomic Region Sequence (DICOM tag: 0008,2218), Anatomic Region Modifier Sequence (DICOM tag: 0008,2220), Primary Anatomic Structure Sequence (DICOM tag: 0008,2228), and Primary Anatomic Structure Modifier Sequence (DICOM tag: 0008, 2230). These were first implemented in the XA Radio-Fluoroscopy, Nuclear Medicine, and Ultrasound Information Objects. The CAP in partnership with ACR-NEMA has created a SDM containing the subset of SNOMED that is referenced by DICOM data elements. The SDM also records additional semantic properties,

$\Varangle$ The triplet encoding technique was invented by Clement J. McDonald, MD for use in the ASTM E-1238 and HL7 Standards. In 1993, triplet encoding was selected by the ANSI HISPP MSDS as the common data type for coded entries. One of the authors (W.D.B.) proposed the inclusion of triplet encoding in a preballot draft of DICOM. The semantics of the original ASTM/HL 7 triplet group were extended and implemented using the DICOM sequence data type. The hybrid construction was named a "triplet encoded sequence." With the assistance of Charles Parisot, many of the original nonsequence-encoded draft DICOM data elements were converted to triplet encoded sequences in time for final ballot of DICOM in December 1993 Subsequent DICOM Supplements routinely use triplet encoded sequences to represent new concepts that are required to build messages for new subject areas. 
such as references to UMLS unique concept identifiers and term-modifier relationships.

HL7-DICOM interface development. The HL7 Standard is the most widely implemented data interchange (message) standard for nonimage patient record information. ${ }^{35}$ The content of the HL7 Standard is closely related to the CEN/TC251/WG3 PT3-022 draft standard for request and report messages for diagnostic service departments. ${ }^{36}$ The classes and attributes of the PT3-022 domain information models are compatible with models being developed by HL7. Because neither the HL7 V.2.2 Standard nor the PT3-022 V.1.1 First Working Document supports diagnostic image communication, both organizations have interest in aligning their standards to be compatible with the
DICOM (and MEDICOM) Standards. ACRNEMA has similar interest in achieving full compatibility with HL7 and PT3-022 in the area of the ISIS Interface. ACR-NEMA and HL7 have therefore formed an HL7 Image Management Special Interest Group (IMSIG) in September 1995, to pursue joint development of the ISIS interface. The agenda of the HL7 Image Management group includes refinement of the ISIS Context Model, mapping of HL7 v.2.x messages to DICOM messages, and convergence of the reference data models of ACR-NEMA DICOM and HL7 Version 3.x. The goal of both organizations is to reference the same reference data model and to specify semantically equivalent messages for the ISIS interface in HL7 V.3.x and in future versions of DICOM. ${ }^{37}$

\section{REFERENCES}

1. Bidgood WD Jr, Horii SC: Introduction to the ACRNEMA DICOM Standard. Radiographics 12:345-355, 1992

2. Horii SC, Bidgood WD Jr: Network and ACR-NEMA Protocols. Radiographics 12:537-548, 1992

3. Digital Imaging and Communications in Medicine (DICOM). NEMA PS 3.1-PS 3.12. Rosslyn, VA, The National Electrical Manufacturers Association 1992-1995.

4. American College of Radiology, National Electrical Manufacturers Association (ACR-NEMA) Standards Publication number 300-1988 (ed 3): Digital Imaging and Communications. Washington, DC, National Electrical Manufacturers Association, 1989

5. Digital Imaging and Communications in Medicine (DICOM). X-Ray Angiographic Image Objects and Media Storage. Rosslyn, VA, The National Electrical Manufacturers Association, 1995 (suppl 4)

6. Digital Imaging and Communications in Medicine (DICOM). Supplement for Endoscopic Image Objects and Media Storage. Text for Public Review. Endoscopic Image Exchange Ad Hoc Committee. ASGE-ACR-NEMA. Rosslyn, VA, National Electrical Manufacturers Association, 1995

7. ACR-NEMA DICOM (American College of Radiology, National Electrical Manufacturers Association Digital Imaging and Communications Standards Committee). Washington, DC, Secretariat: NEMA, 1996

8. CEN/TC251 Medical Informatics Secretariat: BINIBN. Ghent, Belgium, 1995

9. Minutes of CEN/TC251/WG4: Medical Imaging and Multimedia. Brussels, June 19-20 1995. (Adoption of a subset of the ISIS Concept Model as the Domain Information of CEN/TC251/PT4-020: IS-Modality Interface Project Team.) CEN/TC251 Medical Informatics Secretariat: BINIBN. Ghent, Belgium, 1995

10. Minutes of the Joint Meeting of ACR-NEMA WG9 and HL7. Atlanta, GA, May 17-18 1995. Ann Arbor, MI, Health Level Seven, Inc. 1995

11. Minutes of CAP-IEC: College of American Patholo- gists Image Exchange Committee. November 12, 1994. January 3-4 1995, March 18-19 1995, and June 3-4, 1995. Waukegan, IL, College of American Pathologists, 1995

12. EIMEX: Endoscopic Image Exchange Committee. American Society for Gastrointestinal Endoscopy, 1995

13. MIPS: Type 2 Medical Image Processing System Standardization (for Data Interchange over Medical Networks). Tokyo, Japan, Japan Industries Association of Radiation Apparatus, 1995

14. JAHIS: Japanese Association for Healthcare Information Systems. Tokyo. (W.D.B.; Personal Communication, June 21, 1995, Toyoda K. Vice chairman, Executive Division).

15. ISAC: Image Save and Carry Standard. Tokyo, Japan, Medical Information Development Center, July 13, 1995

16. Request and report messages for diagnostic service departments. Medical Informatics CEN/TC 251/N95-027 Draft First Working Document (Red Cover Procedure) CEN/TC 251/PT3-022 BC-IT-M-021, vol 1.1, 1995-08-18

17. National Health Information Modeling Project: Canberra, Australia, The Australian Institute of Health and Welfare, 1995

18. Minutes of the Joint Meeting of ACR-NEMA WG9 and HL7. Atlanta, GA, May 17-18, 1995. Ann Arbor, MI, Health Level Seven Inc, 1995 (ISIS model accepted as the basis for future joint development work.)

19. Minutes of the CAP-IEC. New York, NY, June 3-4, 1995. Northfield, IL, College of American Pathologists, 1995 (ISIS model adopted for standardization of all Pathology imaging modalities.)

20. Minutes of the CEN/TC251/WG4, Brussels, Bel. gium, June 19-20, 1995. CEN/TC251 Medical Informatics Secretariat: BIN-IBN. Ghent, Belgium, 1995, (A subset of the ISIS model adopted as the Domain Information Model of PT4-020 for the Imaging System-Modality interface.)

21. Minutes of CEN/TC251/WG4 June 19-20, 1995. CEN/TC251 Medical Informatics Secretariat: BIN-IBN. Ghent, Belgium, 1995, (Resolution to CEN/TC251 WG3 
indicating the consensus of WG4 Medical Imaging and Multimedia on the request/report concepts of the ISIS model.)

22. Bidgood WD Jr: Invited Presentation on the ISIS Project: "Report on study of commonalities between HL7, DICOM, WG4 and WG3." Lund, Sweden, June 17, 1995. Minutes of CEN/TC251/WG3. Lund, Sweden, June 17-18, 1995. CEN/TC251 Medical Informatics Secretariat: BINIBN, Ghent, Belgium, 1995

23. Bidgood WD Jr: Comments on: TC251 PT3-022 Request and report messages for diagnostic service departments Red Cover Document. In document TC251 WG3 N218. Minutes of CEN/TC251/WG3. Lund, Sweden. June 17-18, 1995. CEN/TC251 Medical Informatics Secretariat: BIN-IBN. Gient, Belgium, 1995

24. Bidgood WD Jr: Invited Presentation on the ISIS Project: "Report on study of commonalities between HL7, DICOM, WG4 and WG3." AHCPR Working Meeting between ANSI HISPP and CEN/TC251 WG1: Medical Records and Modeling. Boston, MA, June 21, 1995. Fitzmaurice $M$ (ed): Report of the Working Meeting between ANSI HISPP and CEN/TC251 WG1: Medical Records and Modeling. Agency for Health Care Policy and Research. Washington, DC, 1995, (in press)

25. Markwell DA: CEN TC251 PT3-022 Alignment with TC251 WG4 and ISIS. July 27, 1995. IST/35/-4/210 Document P22ALIGN.DOC. Birmingham, UK, NHS Information Management Center, British Institute of Standards and Technology.

26. Digital Imaging and Communications in Medicine (MEDICOM/DICOM): Basic Worklist Management (Modality Worklist Management SOP Class). Text for Public Review. Rosslyn, VA, The National Electrical Manufacturers Association, 1995 (suppl 10)

27. Digital Imaging and Communications in Medicine (MEDICOM/DICOM). Storage Commitment Service Class. Text for Letter Ballot. Rosslyn, VA, The National Electrical Manufacturers Association, 1995 (suppl 8)

28. American College of Cardiology: Digital interchange standard for cardiology (DISC) '95. Distributed as a CD at the 44th Annual Scientific Session of the ACC meeting. New Orleans, LA, March 19-22, 1995

29. Bidgood WD Jr, Tracy W: In Search of the Name. Proceedings of the Seventeenth Annual Symposium on Computer Applications in Medical Care. October 30November 3, 1993. American Medical Informatics Association. New York, NY, McGraw-Hill, pp 54-58

30. ANSI HISPP MSDS Common Data Types for Harmonization of Communications Standards in Medical Informatics. Bidgood WD Jr (ed): ANSI HISPP Message Standards Developers Subcommittee. October 30, 1993, (Reprinted in full in the Minutes of the ANSI HISPP MSDS Joint Working Group for Common Data Types. May 19, 1995. Atlanta, GA, Health Level Seven, Inc. Ann Arbor, MI, 1995

31. Parsons DM, Kim Y, Haynor DR: Quality control of cathode-ray tube monitors for medical imaging using a simple photometer. J Digit Imaging 8:10-20, 1995

32. Hemminger BM, Johnston RE, Rolland JP, et al: Introduction to perceptual linearization of video display systems for medical image presentation. J Digit Imaging 8:21-34, 1995

33. Lindberg DAB, Humphreys BL, McCray AT: The Unified Medical Language System. Methods of Information in Medicine 32:281-291, 1993

34. Medical subject headings. Bethesda, MD, National Library of Medicine, 1995

35. Health Level Seven: An Application Protocol for Electronic Data Exchange In Healthcare Environments. Version 2.2, Ann Arbor, MI, Health Level Seven, Inc., 1994

36. Request and report messages for diagnostic service departments. Medical Informatics CEN/TC 251/N95-027 Draft First Working Document (Red Cover Procedure) CEN/TC 251/PT3-022 BC-IT-M-021 vol 1.1. 1995-08-18

37. Minutes of the Joint Meeting of HL7, ACR-NEMA WG9, CEN/TC251 WG4 and CAP-IEC. Kansas City, MO, September 28, 1995, Ann Arbor, MI, Health Level Seven, Inc., 1995 